\section{A Grammastola spatulata mechanotoxin-4 (GsMTx4)-sensitive cation channel mediates increased cation permeability in human hereditary spherocytosis of multiple genetic etiologies}

Hereditary spherocytosis (HS) is the most common inherited hemolytic anemia among people of Northern European descent. HS is caused by mutations in genes encoding the erythroid cytoskeleton proteins ankyrin-1 (ANK1), $\beta$-spectrin (SPTB), and $\alpha$-spectrin (SPTA1), the major intrinsic erythroid membrane protein and chloridebicarbonate exchanger, SLC4A1/band 3, and rarely EPB42/protein 4.2. These mutations lead to destabilization and progressive loss of red cell membrane lipids and surface area, and in some cases to destabilization of cytoskeletal-membrane attachment. The resulting red cells often exhibit normochromic or hyperchromic, mildmoderate microcytosis with increased incubated osmotic fragility and reduced deformability. Anemia and chronic hemolysis can be accompanied by hyperbilirubinemia and painful splenic enlargement. Splenectomy often provides symptomatic relief and attenuates anemia and hemolysis. $^{1}$

Increased erythroid cation permeability in HS was first reported by Harris and Prankerd (1953) and Bertles (1957), as subsequently cited by Zarkowsky et al. ${ }^{2}$ Later reports of increased red cell cation permeability appeared in the setting of Southeast Asian ovalocytosis and cyrohydrocytosis in association with SLC4A1 mutations, in the setting of overhydrated stomatocytosis in association with mutations in RHAG, SLC2A1, and SLC4A1, and in the setting of familial pseudohyperkalemia associated with $A B C B 6$ mutations. ${ }^{3}$ Spherocytic mouse red cells genetically lacking EBP41 or EBP42, or haploinsufficient for SLC4A1 exhibited enhanced Gardos channel activity and increased hemolysis in the presence of the Gardos inhibitor, clotrimazole, ${ }^{4}$ consistent with enhanced nonspecific cation permeability associated with these mouse HS models. Human HS red cells of diverse genotype were uniformly characterized by increased steady-state con-

Table 1. Hereditary spherocytosis mutations accompanied by increased cation currents.

\begin{tabular}{|c|c|c|c|c|c|c|c|c|c|c|}
\hline Subject & Genetic diagnosis ${ }^{\#}$ & Ref. & $\begin{array}{c}\text { Sanger } \\
\text { sequence }\end{array}$ & NPo & $\begin{array}{c}\text { NPo } \\
\text { + GsMTx4 }\end{array}$ & $\begin{array}{l}\text { Unitary } \\
\text { Conductance }{ }^{\mathrm{a}}\end{array}$ & $\begin{array}{l}\text { Fam } \\
H x^{b}\end{array}$ & $\begin{array}{l}\text { Osm } \\
\text { Frge }\end{array}$ & $\begin{array}{l}\mathrm{TX} / \\
\mathrm{Ac}^{\mathrm{d}}\end{array}$ & $\begin{array}{l}\text { SpX/ } \\
C x^{e}\end{array}$ \\
\hline HS1 (M) & $\begin{array}{l}\text { SLC4A1 E68X, } \\
\text { c.202G > T w NMD } \%\end{array}$ & novel ${ }^{\mathrm{f}}$ & cDNA, gDNA & n.d. & n.d. & n.d. & n.a & n.a. & n.a. & n.a. \\
\hline HS2 (F) & $\begin{array}{l}\text { SLC4A1 R150X, c.448C>T, } \\
\text { Band } 3 \text { Lyon w NMD } \%\end{array}$ & (g) & cDNA, gDNA & $\begin{array}{c}1.43 \\
(n=2)\end{array}$ & $\begin{array}{c}0.14 \\
(n=1)\end{array}$ & $35 \mathrm{pS}$ & + & n.a & - & + \\
\hline $\begin{array}{l}\text { HS3, HS4 } \\
\text { (M,F; sibs) }\end{array}$ & $\begin{array}{l}\text { SLC4A1 R490C, c.1648C >T, } \\
\text { Band } 3 \text { Bicetre }\end{array}$ & (h) & cDNA & $\begin{array}{c}3.54 \\
(n=1)\end{array}$ & n.d. & $28 \mathrm{pS}$ & + & + & - & + \\
\hline HS5 (F) & $\begin{array}{l}\text { SLC4A1 M663del, c.1987-9del*, } \\
\text { Band } 3 \text { Osnabruck II }\end{array}$ & (i) & gDNA & $\begin{array}{c}0.91 \pm 0.32 \\
(n=4)\end{array}$ & n.d. & $25 \mathrm{pS}$ & + & + & + & - \\
\hline HS6 (F) & $\begin{array}{l}\text { SLC4A1 R870W, c.2608C>T, } \\
\text { Band } 3 \text { Prague III }\end{array}$ & (j) & cDNA & n.d. & n.d. & n.d. & + & $+/-$ & - & - \\
\hline HS7 (F) & $\begin{array}{l}\text { ANK1 E883Gfs32X, } \\
\text { c.2648delA, w NMD } \%\end{array}$ & novel ${ }^{k}$ & cDNA, gDNA & n.d. & n.d. & n.d. & + & n.a. & + & + \\
\hline HS8 (F) & $\begin{array}{l}\text { A1110-Q1111del, } \\
\text { c.3328-3333del6, w pNMD }{ }^{\% \%} \\
\text { (Exon } 28 \text { mutant alters } \\
\text { splice acceptor site) }\end{array}$ & novel & cDNA, gDNA & $\begin{array}{c}0.56 \pm 0.13 \\
(n=4)\end{array}$ & $\begin{array}{r}0.059 \\
(\mathrm{n}=1)\end{array}$ & $22.5 \mathrm{pS}$ & + & + & + & + \\
\hline HS9 (M) & $\begin{array}{l}\text { ANK1 K1140Gfs86X, } \\
\text { c.3416ins16, w pNMD } \% \%\end{array}$ & novel ${ }^{1}$ & cDNA, gDNA & $\begin{array}{c}0.93 \pm 0.17 \\
(n=4)\end{array}$ & n.d. & $25.8 \mathrm{pS}$ & + & + & - & - \\
\hline HS10 (F) & $\begin{array}{l}\text { ANK1 E1289Gfs86X, } \\
\text { c.3865dupG, w NMD"\% }\end{array}$ & novel & cDNA, gDNA & n.d & n.d. & n.d. & + & + & + & + \\
\hline HS11 (F) & SPTB R1255G, c.3763A>G & $\begin{array}{l}\text { novel variant, } \\
\text { ikely pathogenic }\end{array}$ & gDNA & $\begin{array}{c}1.26 \\
(n=1)\end{array}$ & $\begin{array}{c}0.042 \pm 0.02 \\
(n=4)\end{array}$ & $21 \mathrm{pS}$ & + & n.a. & - & - \\
\hline HS12 (M) & $\begin{array}{l}\text { SPTB G1450Rfs40X, } \\
\text { c.4346dupG, w NMD\% }\end{array}$ & novel & cDNA, gDNA & n.d. & n.d. & n.d. & + & + & + & - \\
\hline HS13 (M) & $\begin{array}{l}\text { SPTB E1815Pfs90X, } \\
\text { c.5443G>CC, w NMD }{ }^{\%}\end{array}$ & novel & cDNA, gDNA & n.d. & n.d. & n.d. & - & + & + & + \\
\hline
\end{tabular}

" CDNA numbering from initiator ATG of the open reading frame; SLC4A1: NP000333.1, NM000342.4; ANK1 isoform 1: NP065209, NM020476.2; SPTA1: NP003117, NM003126.4; SPTB erythrocyte isoform A: NP 001020029.1; var. 1 NM001024858. ${ }^{\circ}$ complete nonsense-mediated decay (NMD); \% partial nonsense-mediated decay (pNMD); ${ }^{*}$ deletion of any three consecutive nucleotides between c.1987-1992; n.d.: not done; n.a.: not available. ${ }^{a}$ Unitary slope conductance measured in a single representative cell of specified genotype, without GsMTx4 in the pipette solution. ${ }^{\mathrm{b}} \mathrm{FamHx}$ : family history of hereditary spherocytosis; ${ }^{\mathrm{c}} \mathrm{OsmFrg}$ : results of osmotic fragility test; ${ }^{\mathrm{d}} \mathrm{Tx} / \mathrm{AC}$ : history of transfusion without or with aplastic crisis; ${ }^{\mathrm{e}} \mathrm{Spx} / \mathrm{Cx}$ : history of splenectomy or cholecystectomy. ${ }^{\mathrm{f}}$ Found with SLC4A1 R180H, c.539G $>\mathrm{A}$, rs 147390654, MAF 0.0001-0.01 in different populations, detected in cDNA and gDNA. ${ }^{\mathrm{g}}$ Alloisio N et al. Blood 1996;88:1062-1069, conallelic with SLC4A1 E40K, c.118G>A, Band 3 Montefiore. Rybicki AC et al. Blood 1993;81:2155-2165. Detected in cDNA and gDNA. ' Dhermy D et al. Br J Haematol 1997;98:32-40. 'Eber SW et al. Nat Genet 1996;13:214-218. Jarolim P et al. Blood 1995;85:634640 , found together with benign variant SLC4A1 K56E, c.166A>G, Band 3 Memphis. Yannoukakos D et al. Blood 1991;78:1117-1120. Detected in cDNA and gDNA. ${ }^{\text {FFound }}$ together with SPTA1 R1074H, c.3221G >A, rs551084590, MAF 0.00004, detected in gDNA. Found together with SLC4A1 P854L, c.2561C >T; and K56E, c.166A>G, Band 3 Memphis II, Bruce LJ et al. J Biol Chem 1994;269:16155-16158. Detected in cDNA. ${ }^{\mathrm{m}}$ Found together with likely benign variant ANK1 R619H, c.1856G>A, rs2304877, Ankyrin Bruggen, Nakanishi $\mathrm{H}$ et al. Int J Hematol 2001;73:54-63. Detected in gDNA. 
A

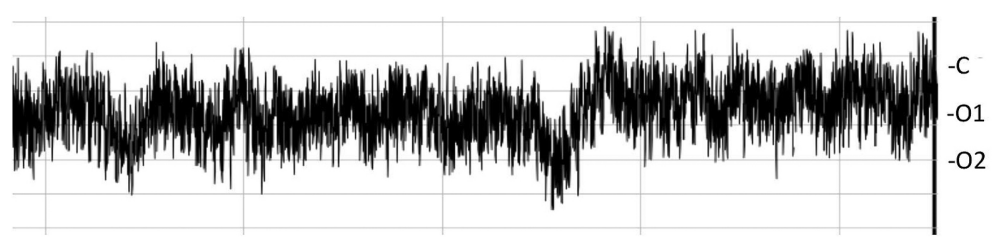

B

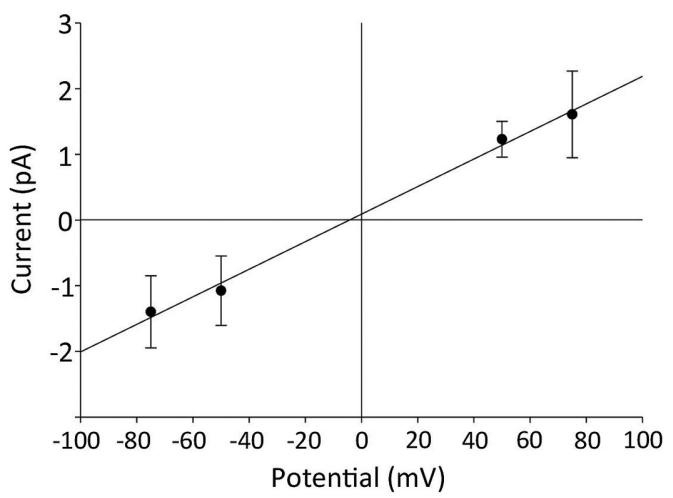

C

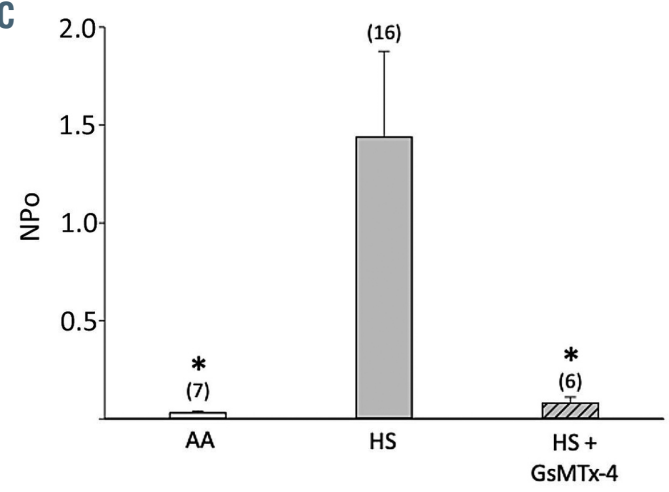

Figure 1. Electrophysiological properties of cation channels in hereditary spherocytosis red cells. (A) A representative current trace recorded at $-\mathrm{Vp}=-25 \mathrm{mV}$ from an on-cell patch recording from a red cell of patient HS11 with hereditary spherocytosis (HS) carrying the novel heterozygous SPTB missense variant R1255G (Polyphen-2 score 0.999). Identical bath and pipette fluid composition included (in mM) $140 \mathrm{NaCl}, 4 \mathrm{KCl}, 1 \mathrm{CaCl}, 1 \mathrm{MgCl}, 10 \mathrm{NaHEPES}$ at a final pH of 7.40. On-cell patch currents were recorded by an Axopatch 200b amplifier and digitized by a Digidata 1440A A-D converter (Molecular Devices, Sunnyvale, CA, USA). Seal resistances were $6.0 \pm 1.0 \mathrm{G} \Omega(n=7)$ in non-HS cells, $5.0 \pm 0.8 \mathrm{G} \Omega(n=14)$ in HS cells without GsMTx4 in the pipette solution, and $4.8 \pm 1.0 \mathrm{G} \Omega$ $(n=6)$ in HS cells with GsMTx4 in the pipette solution. Seal duration for recordings on HS cells unexposed to GsMTx4 was $18 \pm 11$ min. Data were filtered at 500 $\mathrm{Hz}$, digitized at $2 \mathrm{kHz}$ by PClamp and analyzed offline by Clampfit (PCLAMP11, Molecular Devices). (B) Current-voltage relationship of HS11 red cell current measured in a representative on-cell patch, with unitary slope conductance of $21 \mathrm{pS}$. The current-voltage (I-V) relationship was generated in Clampex (PCLAMP 11, Axon Instruments) with the real-time control window in gap-free mode to record current traces of 10-30 s duration. Test potentials were selected in $25-50 \mathrm{mV}$ increments ranging between a minimum of $-100 \mathrm{mV}$ to a maximum of $+100 \mathrm{mV}$. (C) Summary data for NPo calculated from on-cell patch current traces of 5 $10 \mathrm{~s}$ duration recorded in 16 cells from six HS mutant genotypes and in six cells from three mutant HS genotypes in the additional presence of GsMTx4 (1 $\mu \mathrm{M}$ ) in the pipette fluid. NPo values recorded in seven non-HS red cells from four normal individuals (AA) are also shown. $* P<0.05$ for the $t$-test comparing normal to HS cells, and for the Mann-Whitney test comparing HS cells in the presence versus absence of GsMTx4.

centrations of fluorometrically measured intracellular $\left[\mathrm{Ca}^{2+}\right] .{ }^{5}$ However, Petkova-Kirova et al. ${ }^{6}$ recently reported that HS red cells of the same individuals had a spectrum of decreased, increased, or unchanged cation channel activities as measured by an automated whole cell patch clamp technique.

These studies led us to investigate whether HS red cells might be characterized by increased cation channel activity as detected by on-cell patch clamp analysis. We isolated DNA and RNA from whole blood of 13 patients with a clinical diagnoses of HS under protocols approved by Investigational Review Boards of Boston Children's Hospital and Beth Israel Deaconess Medical Center. The hematologic indices of the patients' red cells are presented in Online Supplementary Table S1. From the isolated total RNA, we generated complementary DNA (cDNA) for Sanger sequencing of SLC4A1. cDNA and/or genomic DNA (gDNA) from those patients lacking an evident SLC4A1 mutation in blood cDNA was subjected to Sanger sequencing of $A N K 1$ and SPTB. Whole exome sequencing was reserved for gDNA from the six of 13 patients' samples that remained uninformative. Mutations detected by whole exome sequencing were subsequently confirmed by Sanger sequencing (Table 1). We found seven novel pathogenic mutations and one novel missense variant of very high predicted pathogenicity in previously identified HS genes among these patients with clinical diagnoses of HS. A subset of HS mutant red cells was subjected to on-cell patch clamp analysis (Figure 1). All cells in which stable gigohm seals were achieved exhibited substantial cation channel activ- ity as compared to non-HS red cells. Mean cation channel unitary conductance among HS red cells was $26 \pm 2.1 \mathrm{pS}$ ( $n=6$ genotypes encompassing 16 cells; see Table 1 ). This increased activity, in the cases tested, was nearly completely inhibited by the mechanosensitive cation channel inhibitor, Grammastola spatulata mechanotoxin-4 (GsMTx4) at a concentration of $1 \mu \mathrm{M}$ in the recording pipette (Table 1, Figure 1C). Non-HS red cells from healthy donors exhibited minimal channel activity (Figure 1C), as we had previously reported. ${ }^{7}$

In this collection of HS patients, we found mutations in SLC4A1, ANK1, SPTA1, and SPTB (Table 1). Several patients exhibited mutations in SLC4A1 previously reported in HS. HS2 was heterozygous for both HS mutant Band 3 Lyon (SLC4A1 R150X) and Band 3 Montefiore (SLC4A1 E40K). Each mutation was undetected in cDNA but confirmed in gDNA, strongly suggesting that the mutant transcript carrying both mutations was a substrate of nonsense-mediated mRNA decay. Siblings HS3 and HS4 were each heterozygous for Band 3 Bicetre (SLC4A1 R490C). HS5 was heterozygous for Band 3 Osnabruck (SLC4A1 del663). HS6 was heterozygous for Band 3 Prague III (SLC4A1 R870W), accompanied by the nonpathogenic Band 3 Memphis I (SLC4A1 E56K).

Our HS patients also revealed a novel mutation in SLC4A1 and several novel mutations in ANK1 and SPTB, including a novel SPTB missense variant strongly predicted to be pathogenic (Table 1). The novel SLC4A1 E68X mutation in HS1 was associated with nonsense-mediated decay, whereas the known rare SLC4A1 R180H variant 
found on the other allele was detectable in both cDNA and gDNA. Four HS patients exhibited novel, heterozygous ANK1 loss-of-function mutations, including ANK1 E883Gfs32X in HS7 (accompanied by the known, rare SPTA1 R1074H variant of uncertain significance), ANK1 A1110del2 in HS8 (mutating a splice acceptor site), ANK1 K1140Gfs86X in HS9 (accompanied by the SLC4A1/Band 3 Memphis II polymorphism) and ANK1 E1289Gfs86X in HS10. Combined cDNA and gDNA sequencing indicated that mRNA encoding both ANK1 mutations E883Gfs32X and E1289Gfs76X were substrates of nonsense-mediated decay, whereas the other two ANK1 mutations underwent partial nonsense-mediated decay (Table 1).

Two HS patients were found to have novel heterozygous loss-of-function mutations in the SPTB gene encoding $\beta$-spectrin, SPTB G1450Rfs41X in HS12 and SPTB E1815Pfs90X in HS13 (Table 1). Both of these frameshift termination mutations encoded substrates of nonsensemediated decay. Patient HS11 exhibited compound heterozygosity for the novel, "probably damaging" missense variant SPTB R1255G (Polyphen-2 score 0.999) and the known non-pathogenic ANK1 R619H variant. The likely pathogenic SPTB R1255G substitution is located in the ninth of $\beta$-spectrin's 17 repeat domains, portions of which comprise a dimerization domain, a tetramerization domain, and the ankyrin-binding domain. Remarkably, the purified recombinant ninth $\beta$-spectrin repeat generated in $E$. coli was found to be more unstable (with a melting temperature of $20^{\circ} \mathrm{C}$ ) than any other recombinant $\beta$-spectrin repeat polypeptide, each of which had melting temperatures $\geq 37^{\circ} \mathrm{C},{ }^{8}$ demonstrating increased mutation-associated susceptibility to dysfunctional conformational change.

We assessed some of the HS mutants shown in Table 1 for cation channel activity in on-cell patches, preserving any regulatory components of the red cell cytosol and membrane cytoskeleton. Red cells from patients carrying the known SLC4 HS mutants R150X, R490C, and M663del each exhibited channel activity. Red cells from the patients carrying the novel HS-associated mutations ANK1 A1110del2 and ANK1 K1140Gfs86X also exhibited channel activity. In addition, red cells from the patient carrying the novel, predicted pathogenic VUS SPTB R1255G exhibited channel activity. The representative current trace from patient HS4 in Figure 1A with reversal potential of $\sim 0 \mathrm{mV}$ and unitary conductance of $21 \mathrm{pS}$ (Figure 1B) is consistent with cation channel activity. Oncell patch recordings of red cells from patients HS2, HS4, HS5, HS8, HS9 and HS11, representing mutations in SLC4A1, ANK1, and SPTB, exhibited a mean unitary conductance of $26 \pm 2.1 \mathrm{pS}$.

In on-cell patch recordings of red cells from patients with the previously known SLC4A1 HS mutation R150X (HS2), the novel ANK1 mutation delA1110Q1111 (HS8), and the novel, rare predicted pathogenic SPTB variant R1255G (HS11), channel activity was also monitored under conditions in which the micropipette fluid included the mechanosensitive cation channel blocker, GsMTx4 (1 $\mu \mathrm{M})$. Mean NPo of channel activity was $1.44 \pm 0.44$ as measured in 16 cells representing six genotypes (Figure 1C, Table 1). The presence of $1 \mu \mathrm{M}$ GsMTx 4 in the recording pipette was associated with $\sim 95 \%$ inhibition of channel activity, reducing mean NPo to $0.08 \pm 0.03$ as measured in six cells representing three genotypes (Figure 1C, Table 1). The unitary conductance, reversal potential, and sensitivity of the current to inhibition by GsMTx-4 are each consistent with PIEZO1 mediation of, or contribution to, the measured cation channel activity in HS red cells. The increased membrane tension of the gigaseal inside the pipette ${ }^{9}$ may unmask increased cation current in on-cell patches which might be less readily detected in whole cell patch recordings. ${ }^{5}$ Interestingly, however, small increases in whole cell current were detected in some, if not all HS patients' red cells haploinsufficient for SPTB or for SPTA1.

Cation channel activity in the presence of pathogenic stomatocytosis mutations in transmembrane transporters such as SLC4A1, RHAG, GLUT1, and ABCB6 has been attributed either to direct cation permeation through the dysfunctional mutant membrane protein itself, or to direct or indirect modulation of PIEZO1 activity. ${ }^{10}$ However, the similar properties of the increased cation channel activities measured in the presence of pathogenic HS mutants of the cytoskeletal proteins $\beta$-spectrin and ankyrin very likely arise from direct or indirect modulation of PIEZO1 (and/or another unidentified cation channel), possibly by perturbations transmitted through one of the SLC4A1/Band3-nucleated macro-complexes. ${ }^{11}$ This modulation might reflect PIEZO1 properties such as the lower hydrostatic pressure threshold for PIEZO1 activation in on-cell patches of actin cytoskeleton-depleted cellular blebs than in on-cell patches with intact cell cortex, and/or the inhibition by cytochalasin $\mathrm{D}$ of pressureactivated PIEZO1 in on-cell patches of normal cultured cells, and by glass probe-mediated cell indentation as measured by whole cell currents. ${ }^{9}$

Our data suggest that PIEZO1 likely mediates or contributes a major fraction of the incremental cation permeability of HS red cells. Clarification of the relationships between apparent cytoskeletal modulation of erythroid PIEZO1 and PIEZO1 modulation by flow ${ }^{12}$ and by modulation of lateral membrane tension via the ceramidesphingomyelin balance of the red cell membrane $e^{13}$ will require further study.

David H. Vandorpe,,$^{1 *}$ Boris E. Shmukler, ${ }^{1 *}$ Yann Ilboudo, ${ }^{2}$ Swati Bhasin, ${ }^{3^{\circ}}$ Beena Thomas, ${ }^{3^{\circ}}$ Alicia Rivera, ${ }^{1}$ Jay G. Wohlgemuth, ${ }^{4}$ Jeffrey S. Dlott, ${ }^{4}$ L. Michael Snyder, ${ }^{4}$ Colin Sieff, Manoj Bhasin, ${ }^{3^{\circ}}$ Guillaume Lettre, ${ }^{2}$ Carlo Brugnara ${ }^{6}$ and Seth L. Alper ${ }^{1}$

${ }^{1}$ Division of Nephrology and Vascular Biology Research Center, Beth Israel Deaconess Medical Center and Department of Medicine, Harvard Medical School, Boston, MA, USA; ${ }^{2}$ Montreal Heart Institute and Université de Montréal, Montréal, Québec, Canada; ${ }^{3}$ Division of Integrative Medicine and Vascular Biology Research Center, Beth Israel Deaconess Medical Center and Department of Medicine, Harvard Medical School, Boston, MA, USA; ${ }^{4}$ Quest Diagnostics, Seacaucus, NJ, USA; ${ }^{5}$ Cancer and Blood Disorders Center, Dana-Farber Cancer Center and Boston Children's Hospital, and Department of Pediatrics, Harvard Medical School, Boston, MA USA and 'Department of Laboratory Medicine, Boston Children's Hospital and Department of Pathology, Harvard Medical School, Boston, MA, USA

*DHV and BES contributed equally as co-first authors.

${ }^{\circ}$ Current address: Departments of Pediatrics and Biomedical Informatics, Emory University School of Medicine, Atlanta, GA, USA

Correspondence:

SETH L.ALPER-salper@bidmc.harvard.edu

doi:10.3324/haematol.2021.278770

Received: March 12, 2021.

Accepted: May 28, 2021

Pre-published: June 10, 2021.

Disclosures: JGW and JCD are employees of Quest Diagnostics. $L M S$ and SLA are consultants to Quest Diagnostics. CB has received research funds from Quest Diagnostics after completion of this study. 
Contributions: $D H V, B E S, Y I, S B$, and $B T$ performed research. $D H V, B E S, Y I, S B, B T, A R, M B, G L, C B$, and SLA analyzed data; $C S$ and $C B$ contributed patients; SLA, YI, SB, and $C B$ wrote the paper. All authors reviewed, discussed, and critiqued the paper. SLA supervised the study.

Acknowledgments: we thank Jeff Raddliff (Quest Diagnostics) for editorial suggestions.

Funding: MB was supported by Beth Israel Deaconess Medical Center Core start-up funds. GL was supported by the Canadian Institutes of Health Research (PJT \#156248). YI was supported by University of Montreal faculty of medicine merit scholarships for graduate and postdoctoral studies. SLA was supported by research funds from the Doris Duke Charitable Foundation and from Quest Diagnostics.

\section{References}

1. Iolascon A, Andolfo I, Russo R. Advances in understanding the pathogenesis of red cell membrane disorders. Br J Haematol. 2019; 187(1):13-24

2.Zarkowsky HS, Oski FA, Sha'afi R, Shohet SB, Nathan DG Congenital hemolytic anemia with high sodium, low potassium red cells. I. Studies of membrane permeability. N Engl J Med. 1968; 278(11):573-581

3. Andolfo I, Russo R, Gambale A, Iolascon A. Hereditary stomatocytosis: an underdiagnosed condition. Am J Hematol. 2018;93(1);107121.
4. De Franceschi L, Rivera A, Fleming MD, et al. Evidence for a protective role of the Gardos channel against hemolysis in murine spherocytosis. Blood. 2005; 106(4):1454-1459.

5. Hertz L, Huisjes R, Llaudet-Planas E, et al. Is increased intracellular calcium in red blood cells a common component in the molecular mechanism causing anemia? Front Physiol. 2017;8:673.

6. Petkova-Kirova P, Hertz L, Danielczok J, et al. Red blood cell membrane conductance in hereditary haemolytic anaemias. Front Physiol. 2019; 10:386.

7. Vandorpe $\mathrm{DH}, \mathrm{Xu} \mathrm{C}$, Shmukler BE, et al. Hypoxia activates a Ca2+permeable cation conductance sensitive to carbon monoxide and to GsMTx-4 in human and mouse sickle erythrocytes. PLoS One. 2010;5:e8732

8. An X, Guo X, Zhang X, et al. Conformational stabilities of the structural repeats of erythroid spectrin and their functional implications. J Biol Chem. 281(15):10527-10532.

9. Cox CD, Bae C, Ziegler L, et al. Removal of the mechanoprotective influence of the cytoskeleton reveals PIEZO1 is gated by bilayer tension. Nat Commun. 2016;7:10366.

10. Flatt JF, Bruce LJ. The molecular basis for altered cation permeability in hereditary stomatocytic human red blood cells. Front Physiol. 2018;9:367.

11. Burton NM, Bruce LJ. Modelling the structure of the red cell membrane. Biochem Cell Biol. 2011;89(2):200-215.

12. Evans EL, Povstyan OV, De Vecchis D, et al. RBCs prevent rapid PIEZO1 inactivation and expose slow deactivation as a mechanism of dehydrated hereditary stomatocytosis. Blood. 136(1):140-144.

13. Shi J, Hyman AJ, De Vecchis D, et al. Sphingomyelinase disables inactivation in endogenous PIEZO1 channels. Cell Rep. 2020; 33:108225 